\title{
Study on GM(1,1) Boundary Model based on Surveying Engineering
}

\author{
Yonghe Deng
}

Department of Civil Engineering,Lishui College,Lishui 323000,China

Isxydengyonghe@sina.cn

Keywords: $\mathrm{GM}(1,1)$; boundary condition;safety monitorin;surveying engineering

\begin{abstract}
GM(1,1) models are widely applied to many fields including surveying engineering. Their researches are growing,and study on boundary conditions of $\operatorname{GM}(1,1)$ models is an important work.Based on least squares method,two sorts of new and better methods about boundary condition of $\operatorname{GM}(1,1)$ models were put forward in this paper.Finally,author proved that some models are equals,and proposed some advices.These results are important to $\mathrm{GM}(1,1)$ models and distortion monitoring of surveying engineering .
\end{abstract}

\section{Introduction}

Because GM $(1,1)$ model is simple, and it has higher prediction accuracy based on a few samples ,so,it has been widely used in surveying engineering such as high slope safety monitoring, building settlement prediction, prediction of dam, landslide monitoring, etal[1-10], and has been continually developed.Currently,we watched researches of $\operatorname{GM}(1,1)$ are ascending,and boundary condition of $\mathrm{GM}(1,1)$ is an important research direction.

At first,boundary condition of GM $(1,1)$ has been selected as $\hat{X}^{(1)}(1)=X^{(1)}(1)$ [10], but prediction accuracy of $\operatorname{GM}(1,1)$ is not high, because $X^{(1)}(1)$ is the oldest data, so,some people have selected the newest data as boundary value and $\hat{X}^{(1)}(n)=X^{(1)}(n)$ as boundary condition[11,12,16]. But people found $\hat{X}^{(1)}(n)=X^{(1)}(n)$ as boundary condition is still not good,so they have selected the best con dition among $\hat{X}^{(1)}(m)=X^{(1)}(m)(m=1,2, \ldots, n)$ as boundary condition[1,13,14].For error of the boundary condition $\hat{X}^{(1)}(1)=X^{(1)}(1)$, someone selected $\hat{X}^{(1)}(1)=X^{(1)}(1)+b$ as boundary condition to improve accuracy[15]; but literature[2] directly selected least squares method to improve accuracy.

The results of these studies undoubtedly enriched and developed GM $(1,1)$ boundary condition theory.Based on previous studies, author proposed two new and better boundary models of GM(1,1),verified validity of these new models, proved some models are equals,these works have a positive significance of theory and practice for GM $(1,1)$ model and deformation monitoring of surveying engineering .

\section{Two new and improved boundary models of GM(1,1) put by author}

It is obvious that,because of considered least squares method,model $\hat{X}^{(1)}(1)=X^{(1)}(1)+b$ is superior to model $\hat{X}^{(1)}(1)=X^{(1)(1)}$ [15]; and because of considered the newest information,model $\hat{X}^{(1)}(n)=X^{(1)}(n)$ is superior to model $\hat{X}^{(1)}(1)=X^{(1)}(1)[11,12,16]$. But literatures[15,11,12,16] only considered one aspect,so they are undoubtedly not perfect. If we take into account both the newest information and least squares method, we can get a new and better model such as $\hat{X}^{(1)}(n)=X^{(1)}(n)+b$ based on least squares method in this paper.

Literatrues[1,13,14] thought the best boundary conditions model among $\hat{X}^{(1)}(m)=X^{(1)}(m)$ $(m=1,2, \ldots, n)$ is better than that of $\hat{X}^{(1)}(n)=X^{(1)}(n)$,but they didn't consider least squares 
method,so,it is more valid that I select the best boundary conditions model among $\hat{X}^{(1)}(m)=X^{(1)}(m)+b_{m}(m=1,2, \ldots, n)$ based on least squares method in this paper.

\section{Forecasting or fitting models and their equivalence among different boundary conditions of GM (1,1)}

Forecasting or fitting model based on $\hat{X}^{(1)}(n)=X^{(1)}(n)+b$ and least squares method

According to literature[13], based on boundary condition as $\hat{X}^{(1)}(n)=X^{(1)}(n)+b$, we can obtain

$\hat{X}^{(1)}(k+1)=\left[X^{(1)}(n)+b-\frac{u}{a}\right] e^{-a(k-n+1)}+\frac{u}{a}$

According to Eq.1 and the minimum of $\sum_{k=0}^{n-1}\left[\hat{X}^{(1)}(k+1)-X^{(1)}(k+1)\right]^{2}$, we can get

$b=-X^{(1)}(n)+\frac{u}{a}+\frac{\left(1-e^{-2 a}\right) e^{a(1-n)}}{\left(1-e^{-2 a n}\right)} \sum_{k=0}^{n-1}\left[X^{(1)}(k+1) e^{-a k}\right]-\frac{u}{a} \frac{e^{a(1-n)}\left(1+e^{-a}\right)}{\left(1+e^{-a n}\right)}$

If we bring Eq.2 to Eq.1, forecasting or fitting model is shown as Eq.3.

$$
\hat{X}^{(1)}(k+1)=\left\{\frac{\left(1-e^{-2 a}\right)}{\left(1-e^{-2 a n}\right)} \sum_{k=0}^{n-1}\left[X^{(1)}(k+1) e^{-a k}\right]-\frac{u}{a}\left(\frac{1+e^{-a}}{1+e^{-a n}}\right)\right\} e^{-a k}+\frac{u}{a}
$$

There is another method to compute forecasting or fitting model as follow. First,according to Eq.1,we can obtain

$$
\hat{X}^{(0)}(k+1)=\left(1-e^{a}\right)\left[X^{(1)}(n)+b-\frac{u}{a}\right] e^{-a(k-n+1)}
$$

Then, according to Eq.4 and the minimum of $\sum_{k=0}^{n-1}\left[\hat{X}^{(0)}(k+1)-X^{(0)}(k+1)\right]^{2}$, we can get

$$
b=-X^{(1)}(n)+\frac{u}{a}+\frac{\left(1-e^{-2 a}\right) e^{a(1-n)}}{\left(1-e^{a}\right)\left(1-e^{-2 a n}\right)} \sum_{k=0}^{n-1}\left[X^{(0)}(k+1) e^{-a k}\right]
$$

If we bring Eq.5 to Eq.4, forecasting or fitting model is shown as Eq.6.

$$
\hat{X}^{(0)}(k+1)=\frac{\left(1-e^{-2 a}\right) e^{-a k}}{\left(1-e^{-2 a n}\right)} \sum_{k=0}^{n-1}\left[X^{(0)}(k+1) e^{-a k}\right]
$$

Forecasting or fitting model based on the best model among $\hat{X}^{(1)}(m)=X^{(1)}(m)+b_{m}$ $(m=1,2, \ldots, n)$ and least squares method

According to the best boundary conditions among $\hat{X}^{(1)}(m)=X^{(1)}(m)+b_{m}(m=1,2, \ldots, n)$, we can obtain

$$
\hat{X}^{(1)}(k+1)=\left[X^{(1)}(m)+b_{m}-\frac{u}{a}\right] e^{-a(k-m+1)}+\frac{u}{a}
$$

where: $m$ of Eq.7 is selected the best value among $1,2, \ldots, n$ to make model be best.

According to Eq.7 and the minimum of $\sum_{k=0}^{n-1}\left[\hat{X}^{(1)}(k+1)-X^{(1)}(k+1)\right]^{2}$, we can get

$$
b_{m}=-X^{(1)}(m)+\frac{u}{a}+\frac{\left(1-e^{-2 a}\right) e^{a(1-m)}}{\left(1-e^{-2 a n}\right)} \sum_{k=0}^{n-1}\left[X^{(1)}(k+1) e^{-a k}\right]-\frac{u}{a} \frac{e^{a(1-m)}\left(1+e^{-a}\right)}{\left(1+e^{-a n}\right)}
$$

If we bring Eq.8 to Eq.7, forecasting or fitting model is shown as Eq.9. 


$$
\hat{X}^{(1)}(k+1)=\left\{\frac{\left(1-e^{-2 a}\right)}{\left(1-e^{-2 a n}\right)} \sum_{k=0}^{n-1}\left[X^{(1)}(k+1) e^{-a k}\right]-\frac{u}{a}\left(\frac{1+e^{-a}}{1+e^{-a n}}\right)\right\} e^{-a k}+\frac{u}{a}
$$

There is still another method to compute forecasting or fitting model as follow.First,according to Eq.7,we can obtain

$$
\hat{X}^{(0)}(k+1)=\left(1-e^{a}\right)\left[X^{(1)}(m)+b_{m}-\frac{u}{a}\right] e^{-a(k-m+1)}
$$

Then, according to Eq.10 and the minimum of $\sum_{k=0}^{n-1}\left[\hat{X}^{(0)}(k+1)-X^{(0)}(k+1)\right]^{2}$, we can get

$$
b_{m}=-X^{(1)}(m)+\frac{u}{a}+\frac{\left(1-e^{-2 a}\right) e^{a(1-m)}}{\left(1-e^{a}\right)\left(1-e^{-2 a n}\right)} \sum_{k=0}^{n-1}\left[X^{(0)}(k+1) e^{-a k}\right]
$$

If we bring Eq.11 to Eq.10, forecasting or fitting model is shown as Eq.12.

$$
\hat{X}^{(0)}(k+1)=\frac{\left(1-e^{-2 a}\right) e^{-a k}}{\left(1-e^{-2 a n}\right)} \sum_{k=0}^{n-1}\left[X^{(0)}(k+1) e^{-a k}\right]
$$

Because Eq.9 and Eq.12 do not contain variable $m$,so, whether $m$ selects any value among 1,2, ., $n$, corresponding boundary conditions models are equivalent, their precisions are same.

Forecasting or fitting model based on $\hat{X}^{(1)}(k+1)=-\frac{c}{a} e^{-a k}+\frac{u}{a}$ and least squares method

According toEq.1, we can hypothesize

$$
\hat{X}^{(1)}(k+1)=-\frac{c}{a} e^{-a k}+\frac{u}{a}
$$

According to Eq.13 and the minimum of $\sum_{k=0}^{n-1}\left[\hat{X}^{(1)}(k+1)-X^{(1)}(k+1)\right]^{2}$,we can get

$$
c=-a \frac{1-e^{-2 a}}{1-e^{-2 a n}} \sum_{k=0}^{n-1}\left[X^{(1)}(k+1) e^{-a k}\right]+u\left(\frac{1+e^{-a}}{1+e^{-a n}}\right)
$$

If we bring Eq.14 to Eq.13, forecasting or fitting model is shown as Eq.15.

$$
\hat{X}^{(1)}(k+1)=\left\{\frac{\left(1-e^{-2 a}\right)}{\left(1-e^{-2 a n}\right)} \sum_{k=0}^{n-1}\left[X^{(1)}(k+1) e^{-a k}\right]-\frac{u}{a}\left(\frac{1+e^{-a}}{1+e^{-a n}}\right)\right\} e^{-a k}+\frac{u}{a}
$$

There is another method to compute forecasting or fitting model as follow. First,according to Eq.13,we can obtain

$$
\hat{X}^{(0)}(k+1)=-\frac{c}{a}\left(1-e^{a}\right) e^{-a k}
$$

Then, according to Eq.16 and the minimum of $\sum_{k=0}^{n-1}\left[\hat{X}^{(0)}(k+1)-X^{(0)}(k+1)\right]^{2}$, we can get

$$
c=-a \frac{1-e^{-2 a}}{\left(1-e^{-2 a n}\right)\left(1-e^{a}\right)} \sum_{k=0}^{n-1}\left[X^{(0)}(k+1) e^{-a k}\right]
$$

If we bring Eq.17 to Eq.16, forecasting or fitting model is shown as Eq.18.

$$
\hat{X}^{(0)}(k+1)=\frac{\left(1-e^{-2 a}\right) e^{-a k}}{\left(1-e^{-2 a n}\right)} \sum_{k=0}^{n-1}\left[X^{(0)}(k+1) e^{-a k}\right]
$$

In this paper,because Eq.9 , Eq.15 and Eq.3 are equals, Eq.12 ,Eq.18 and Eq.6 are equals,so, forecasting or fitting models based both on $\hat{X}^{(1)}(n)=X^{(1)}(n)+b$ and least squares method, or based both on the best model among $\hat{X}^{(1)}(m)=X^{(1)}(m)+b_{m}(m=1,2, \ldots, n)$ and least squares method, or 
based both on $\hat{X}^{(1)}(k+1)=-\frac{c}{a} e^{-a k}+\frac{u}{a}$ and least squares method,are equals.

Then, boundary conditions of literature[2] and [15] should bring same result,but literature[2] published in 2009 year thought that it improved boundary condition of literature[15] published in 2001 year before 8 years,this is wrong.

\section{Conclusion}

1) These two new and better models of GM $(1,1)$ boundary condition proposed by author in this paper are feasible and effective.

2) This paper revealed,enriched and developed theoretical relationship of some boundary conditions of GM(1,1) model.

3) In practice of surveying engineering,this paper can help us reduce much computation work because some methods are equals.

4) This paper pointed out there is mistake in literature[15].

5) These results in this paper can be used to all fields of GM(1,1) application.

\section{References}

[1] Tianguo Tang, Xin Wan, Haowu Liu.Improved GM model for safety monitoring of high rock slopes [J].Chinese Journal of Rock Mechanics and Engineering ,2005,24(2):307-312.(In Chinese)

[2] Yongrong Sun ,Yingdong $\mathrm{Hu}$, Wu Chen,etal.Accurate estimation of building deformation based on improved grey GM(1,1) model[J]. Journal of Nanjing University of Aeronautics and Astronautics, 2009,41(1):107-110. (In Chinese)

[3] Hui Yin , Jongwang Ding, Yan Zhang,etal.Integratrd dynamic forecasting by grey numbers of identical dimensions and its application in deformation forecasting[J].Journal of Wuhan Technical University of Surveying and Mapping, 1996,21(1):31-35. (In Chinese)

[4] Yueming Lan Xinzhou,Wang .Research of grey forecast on dam level displacement[J]. Journal of Wuhan Technical University of Surveying and Mapping,1996,21(4):350-354. (In Chinese)

[5] Feng Xie, Luming Zhu , Lizhong Wang . Modified grey system forecasting model and its application for analyzing[J]. Chinese Journal of Rock Mechanics and Engineering,2005, 24(22):4099-4105. (In Chinese)

[6] Yonghe Deng. Study Improved Background Value Functions of Non-equidistance GM(1,1) Model in Surveying and Mapping Engineering[J] .Applied Mechanics and Materials,2011, 90-93:2887-2890.

[7] Yonghe Deng. Improved Combination GM(1,1)Model and Its Application in Distortion Inspction [J] .Applied Mechanics and Materials,2011,99-100:229-232.

[8] Hongran Li, Agen Zhang, Weimin Ye.Accumulating method GM(1,1) model and prediction of land subsidence [J]. Rock and Soil Mechanics,2008, 29(12):3417-3421. (In Chinese)

[9] Yonghe Deng. GM(1,1) model based on least absolute estimation of residual [J] .Applied Mechanics and Materials,2011,90-93 :2881-2886.

[10] Shifan Qiao , Ligang Fang, Baochen Liu.Application of GM(1,1) model and index curve model in the forecast of foundation pile settlement [J].China Railway Science, 2005,26(3):53-55. (In Chinese)

[11] Yungui Li,Qingfu Li,Guofan Zhao.An Improvement on Grey Forecasting Model[J].System Engineering,1992,10(6):27-31. (In Chinese)

[12] Yaoguo Dang,Sifeng Liu,Bin Liu.The GM Models that $\mathrm{x}(1)(\mathrm{n})$ is Taken as Initial Value[J] .Chinese Journal of Management Science,2005,13(1):132-135. (In Chinese)

[13] Dahai Zhang,Shifang Jiang,Kaiquan Shi.Theoretical Defect of Grey Prediction Formula and Its Improvement[J].Systemic Engineering Theory and Practice,2002,(8):140-142. (In Chinese)

[14] Yong Mu.Accurate Socution to Grey Forecasting Model[J]. Journal of Ji'nan University(Science and Technology),2003,17(1):49-50. (In Chinese) 
[15] Hui Zhang,Shigeng Hu.Analysis Boundary Condition for GM(1,1) Model[J].Journal of Huazhong University of Science and Technology, 2001,29(4):110-111. (In Chinese)

[16] Fenyi Dong,Jun Tian.Optimazition Integrated Background Value with Original Condition for GM(1,1)[J].Systems Engineering and Electronics,2007,29(3):464-466. (In Chinese) 